\title{
Totgesagte leben länger
}

\section{Die Wiedergeburt der Sozialen Marktwirtschaft angesichts der globalen Wirtschaftskrise? ${ }^{*}$}

\author{
MiCHAEL S. AßLÄNDER ${ }^{* *}$
}

\section{The Renaissance of Social Market Economy in the Face of the Global Eco- nomic Crisis}

Hayek once called it a weasel-word: The social market economy. Only very recenty, doubts rose again over the conception of social market economy and its ability to cope with the global challenges. However, in the course of the economic crisis the conception has gained increasing importance. Highlighting the political interventions and modifications of social market economy as well as societal processes in recent years the article will demonstrate the threats to social market economy and discuss its possible renaissance.

Keywords: Soziale Marktwirtschaft, Wirtschaftskrise, Finanzkrise, Ordoliberalismus, Wirtschaftsordnung, Gesellschaftsordnung

\section{Einleitung}

Angesichts der aktuellen Finanz- und Wirtschaftskrise wird der Ruf nach staatlichen Interventionen zunehmend lauter. Sich selbst überlassene, weitgehend liberalisierte Märkte, so die schmerzliche Erkenntnis, seien auf Dauer weder in der Lage zu einer optimalen Faktorallokation beizutragen, noch für eine gerechte Güterverteilung zu sorgen (vgl. u. a. Zinn 2009a: 13ff.; Zinn 2009b: 262-275; Hengsbach 2009: 295ff.). War es das Ziel einer neoliberalen Angebotspolitik, durch den Abbau staatlicher Beschränkungen neue Wettbewerbskräfte freizusetzen und so zu Wirtschaftswachstum und Abbau von Arbeitslosigkeit beizutragen, haben sich diese Versprechen angesichts der durch Fehlspekulationen, Selbstüberschätzung der Marktakteure und fehlerhafte Anreizsysteme verursachten wirtschaftlichen Schäden als falsch erwiesen.

Augenscheinlich zwingt die Krise zum Umdenken. Das Konzept der Sozialen Marktwirtschaft, noch vor wenigen Jahren durch die Vertreter einer neoliberaleren Wirtschaftspolitik einstimmig exiliert, soll nun zurückbeordert werden und beim Gesundbeten der Wirtschaftskrise helfen. Dabei verbinden die meisten Politiker bestenfalls vage Vorstellungen mit dem eigentlichen Konzept der ,Sozialen Marktwirtschaft' (vgl.

Beitrag eingereicht am 23.02.2010, nach doppelt verdecktem Gutachterverfahren überarbeitete Fassung angenommen am 01.11.2010.

** Prof. Dr. Michael S. Aßländer, Internationales Hochschulinstitut Zittau, Markt 23, D-02763 Zittau, Tel: +49-(0)3583-61-2774, Fax: +49-(0)3583-61-2785, E-Mail: masslaender@ihi-zittau.de, Forschungsschwerpunkte: Angewandte Ethik, Wirtschaftsethik, Arbeitsbegriff, Theoriegeschichte der Ökonomie. 
Aßländer/Ulrich 2009: 14). Dies ist wenig verwunderlich. Zum Einen präsentiert sich das Konzept der Sozialen Marktwirtschaft bei näherer Analyse selbst keineswegs als einheitliches ,Lehrgebäude‘ (vgl. u. a. Aßländer 2009a: 37f.; Ulrich 2009: 352-356). Selbst bei den Vätern der Sozialen Marktwirtschaft wichen die Vorstellungen, wie viel ,Staat' und wie viel ,Wettbewerb' das geeignete Maß für eine soziale Marktwirtschaft darstellen, durchaus erheblich voneinander ab (vgl. u. a. Müller-Armack 1972: 26; Röpke 1979a: 53; Röpke 1979b: 265f.). Zum Anderen spiegelt sich diese Unschärfe des Konzepts auch in den Reformbemühungen um eine zeitgemäße Anpassung der Sozialen Marktwirtschaft und den jeweils unterschiedlichen ,Krisenrezepturen “ der politischen Akteure wider (vgl. u. a. Quaas 2009: 124-129). ${ }^{1}$

Im folgenden Beitrag soll es jedoch weder darum gehen, die Unterschiede in den theoretischen Entwürfen der Vordenker der Sozialen Marktwirtschaft herauszuarbeiten, noch darum, die aktuelle Tagespolitik des derzeit allgegenwärtigen Kriseninterventionismus zu bewerten. Ob die politischen Maßnahmen erfolgreich waren und welchen Verlauf die Krise noch nehmen wird, lässt sich ohnehin erst aus der Retrospektive erkennen. Vielmehr soll der Frage nachgegangen werden, ob eine Restaurierung der Sozialen Marktwirtschaft und die Rückbesinnung auf ein Primat der Politik in wirtschaftlichen Fragen, wie es als verbindendes Element der theoretischen Entwürfe einer sozial-marktwirtschaftlichen Wirtschaftsordnung gesehen werden kann, als Ordnungsprinzip der Wirtschaft geeignet erscheint, um der Krise entgegenzuwirken und zukünftige Fehlentwicklungen zu vermeiden. Vergessen werden soll dabei nicht, dass sich das Konzept der Sozialen Marktwirtschaft nicht nur als alternativer ,Dritter Weg zwischen reinem Wirtschaftsliberalismus und staatlicher Zentralverwaltungswirtschaft sondern auch als Antwort auf die Krisenerfahrungen der Jahre 1919 und 1929 ve rsteht (vgl. Aßländer 2009b: 228f.). So schreibt Walter Eucken (1990: 309) zu seinem eigenen Konzept der Wettbewerbsordnung:

„Im dritten Jahrzehnt unseres Jahrhunderts liefen die langen Serien konjunkturpolitischer Versuche in den Vereinigten Staaten, Deutschland, England usw. in die Krisenkatastrophe 1929/32 aus. Hier lag der unmittelbare Misserfolg deutlich zutage. - Demgegenüber gelang es zwar den Experimenten der Vollbeschäftigungspolitik und der Politik der zentralverwaltungs-wirtschaftlichen Lenkung, Arbeitslosigkeit zu vermeiden oder vorhandene Arbeitslosigkeit zu beseitigen, aber mit dem Erfolg, dass der Teufel mit dem Beelzebub ausgetrieben wurde. Eine wirtschaftliche Not trat an die Stelle der anderen. Warum scheiterten die konjunkturpolitischen Versuche? Was soll positiv geschehen?"

Um mindestens der letzten der beiden Fragen angesichts der aktuellen Krise aus der theoretischen Perspektive der Sozialen Marktwirtschaft nachzugehen, soll im Folgenden zunächst in der gebotenen Kürze eine Chronologie der aktuellen Ereignisse wiedergegeben (Kapitel 2) und das Konzept der Sozialen Marktwirtschaft in den für die nachfolgenden Betrachtungen wichtigsten Grundzügen erläutert werden (Kapitel 3). In einem weiteren Schritt gilt es, jene Fehlentwicklungen aufzuzeigen, die als Ursache

$1 \quad$ Beleg hierfür ist nicht zuletzt der Richtungsstreit zwischen Frank und Gerhard Maier-Rigaud auf der einen und Hans Willgerodt auf der anderen Seite; zu den unterschiedlichen Positionen vgl. Maier-Rigaud/Maier-Rigaud (2001) und (2009), sowie Willgerodt (2006) und (2007). 
für die aktuelle Finanz- und Wirtschaftskrise genannt werden können (Kapitel 4). Dabei soll vor allem der Frage nach den Fehlentwicklungen in Politik (Kapitel 4.1), Wirtschaft (Kapitel 4.2) und Gesellschaft (Kapitel 4.3) aus sozial-marktwirtschaftlicher Perspektive nachgegangen werden. Schließlich soll abschließend die Zukunftsfähigkeit sozial-marktwirtschaftlicher Konzeptionen vor dem Hintergrund der globalen Finanzund Wirtschaftskrise diskutiert werden (Kapitel 5).

\section{Der Weg in die Wirtschaftskrise - Chronologie der Ereignisse}

Ihren Anfang nahm die derzeitige Wirtschaftskrise mit der so genannten SubprimeHypotheken-Krise auf dem US-Immobilienmarkt im Sommer 2007. Mit dem Begriff Subprime-Hypotheken werden dabei Hypothekendarlehen bezeichnet, die an Schuldner mit geringer Bonität ausgegeben werden. Diese Darlehensnehmer zeichnen sich durch einen bereits relativ hohen Verschuldungsgrad aus - ihr durchschnittlicher Schuldendienst beträgt mehr als 40 Prozent des laufenden Bruttoeinkommens - und sind in mindestens einem Fall ihrem Schuldendienst nicht oder nicht vollständig nachgekommen (vgl. Heine 2007: 2). Auf der in den USA zur Bestimmung der Kreditwürdigkeit üblichen ,FICO Credit Score` werden diese Darlehensnehmer mit weniger als 620 Punkten von 850 erreichbaren bewertet (vgl. Gerardi et al. 2008: 8).

Durch die im Nachgang zu den Ereignissen vom 11. September 2001 eingeleitete Niedrigzinspolitik der amerikanischen Notenbank kam es zu einem allgemeinen günstigen Kreditangebot auf den amerikanischen Märkten. Zahlreiche Hypothekenschuldner nutzten dies, um ihre Darlehen auf günstigere Finanzierungsarten umzuschulden. Da das Zinsniveau teilweise unterhalb der Inflationsrate lag (vgl. Münchau 2008: 6), kam es zudem zu einer Ausweitung der (kreditfinanzierten) Nachfrage nach Wohnimmobilien mit einem allgemeinen Preisanstieg auf dem Immobilienmarkt als Folge (vgl. Bundesverband Deutscher Banken 2009).

Obwohl das Zinsniveau ab 2004 allmählich wieder zu steigen begann und sich das Ausfallrisiko der Banken damit erhöhte, genügte vielen Kreditinstituten das prognostizierte Wachstum auf den Immobilienmärkten als Sicherheit für ihre Hypothekendarlehen. - So prognostizierten etwa die Analysten von Lehman Brothers im Sommer 2005 einen jährlichen Preisanstieg auf den Immobilienmärkten mit einer Wahrscheinlichkeit von 80 Prozent (vgl. Storbeck 2009). - Man ging davon aus, dass es dem Schuldner bei steigenden Immobilienpreisen jederzeit möglich sei, seine Hypothek durch Verkauf der Immobilie zu tilgen (vgl. Storbeck 2009; Bundesverband Deutscher Banken 2009). Zeitgleich führte die allmähliche Sättigung des Hypothekenmarktes dazu, dass Kredite immer häufiger an Kunden mit immer geringerer Bonität vergeben und die Kreditvergabepraktiken der Banken zunehmend riskanter wurden.

Als problematisch erwies sich im Nachgang, dass die Beleihungshöhe der Immobilien im Subprime-Segment durchschnittlich über 80 Prozent lag (vgl. Heine 2007: 2) und bei nahezu der Hälfte aller Kreditnehmer im Jahre 2006 über 90 Prozent des Immobilienwertes erreichte (vgl. Gerardi et al. 2008: 9f.). Dies war vor allem einer Bankenpolitik geschuldet, die durch die Gestaltung zunehmend ,kundenfreundlicherer' Vertragsmodalitäten versuchte, auch einkommensschwache Kunden für das Hypothekengeschäft zu gewinnen. So wurde zum einen die Kreditwürdigkeitsprüfung der Kunden zunehmend laxer gehandhabt (vgl. Gerardi et al. 2008: 8; Storbeck 2009). Zum ande- 
ren wurde versucht, Kunden durch scheinbar günstige Rückzahlungsmodalitäten zu ködern. So wurden beispielsweise Hypothekendarlehen mit 'interest-only-periods angeboten, in denen die Kreditnehmer zunächst nur zur Zinszahlung, nicht aber zur Tilgung verpflichtet waren. Auch lockten die Kreditinstitute mit niedrigen festen Zin ssätzen zu Beginn der Rückzahlungsphase, um im Anschluss daran eine variable Zinsanpassung durchzuführen, was bei steigenden Zinssätzen für viele Hypothekenschuldner mit variablen Zinssätzen den finanziellen Ruin bedeutete (vgl. Endres 2007).

Zunächst schien die Rechnung der Banken jedoch aufzugehen, und das Geschäft mit Hypotheken-Darlehen florierte. Um die stetig wachsende Nachfrage nach Hypothekendarlehen bedienen zu können, begannen die Kreditinstitute, die von ihnen gewährten Hypothekendarlehen in handelbaren Wertpapieren zu verbriefen, um so weitere liquide Mittel zur Kreditvergabe zu generieren (vgl. Münchau 2008: 98-107; Bloss et al. 2009: 71-75; Lin-Hi/Suchanek 2009: 22). Dergestalt gelang es, Hypothekenforderungen aus der Bilanz auszulagern und diese in Form von Finanzderivaten zu handeln, die wiederum durch Bündelung und Tranchierung in unterschiedliche Risikostufen scheinbar mühelos an die Kundenwünsche auf dem Finanzmarkt angepasst werden konnten.

Als Ende 2006 das Zinsniveau der Federal Reserve nach einem allmählichen Anstieg mehr als fünf Prozent erreichte und sich dieses höhere Zinsniveau vor allem bei den Hypothekenschuldnern mit variablen Zinssätzen bemerkbar machte, kam es zu ersten Zahlungsausfällen. Da infolge einer allmählich eingetretenen Marktsättigung und der nun notwendigen Zwangsverkäufe das Preisniveau auf den Immobilienmärkten nachgab, konnten die Zahlungsausfälle nicht mehr durch den Verkauf der Immobilien kompensiert werden, und es kam zu ersten ernsten Verwerfungen innerhalb des ausgeklügelten Systems (vgl. Münchau 2008: 15f.).

Dies wäre bis dahin ein weitgehend endemisches Phänomen geblieben, hätten die von den Banken ausgegebenen Finanzprodukte nicht zwischenzeitlich Einzug in die Wertpapierdepots nahezu aller Großbanken weltweit gehalten. Mit dem Zusammenbruch des US-Immobilienmarktes kam es auch zu massiven Kurseinbrüchen bei den hypothekenbesicherten Finanzderivaten. In der Folge geriet im Juni 2007 die USamerikanische Investmentbank Bear Stearns aufgrund von Hypothekenspekulationen in Schieflage und setzte die Rücknahme von Kapitalanteilen zweier hypothekenlastiger Hedgefonds aus, da sie nach eigenen Angaben nicht mehr in der Lage war, den Wert der in den Fonds gehaltenen strukturierten Finanzprodukte zu ermitteln (vgl. Cohan 2008). Trotz massiver Stützungszahlungen aus eigenen Mitteln war das Bankhaus nicht in der Lage, die Forderungen der Kapitalgeber ihrer beiden Hedgefonds zu bedienen und wurde schließlich von J. P. Morgan Chase übernommen (vgl. Cohan 2008). Zu den ersten Opfern in Deutschland zählten die IKB sowie die Sachsen LB, die sich auf den amerikanischen Hypothekenmärkten verspekuliert hatten. Aufgrund der Schieflage einzelner Banken begann die Furcht vor weiteren Konkursen um sich zu greifen und es kam zu dem, was vielfach als, Vertrauenskrise` auf dem Bankenmarkt bezeichnet wurde (vgl. u. a. Stöttner 2009: 17). Das Misstrauen der Banken untereinander brachte das Interbanken-Geschäft nahezu zum Erliegen, mit erheblichen Liquiditätsengpässen auf den Kapitalmärkten als Folge (vgl. Münchau 2008: 
21ff.; Stöttner 2009: 10). Damit begann sich die Bankenkrise nun auch auf die Realwirtschaft auszuwirken. Um den Liquiditätsengpässen und den durch Wertpapierverluste induzierten Kaufkraftverlusten entgegenzuwirken, sahen sich die Regierungen weltweit dazu veranlasst, den Zusammenbruch des Bankensektors durch massive Stützungszahlungen zu verhindern und einer wirtschaftlichen Rezession durch groß angelegte Konjunkturprogramme entgegenzuwirken.

\section{Das Konzept der Sozialen Marktwirtschaft - Interdependenz der Ordnungen}

Wenn es darum geht, das Konzept der Sozialen Marktwirtschaft zu bestimmen, ergeben sich zunächst zwei Schwierigkeiten. Zum Einen bezeichnet Soziale Marktwirtschaft die real existierende Wirtschaftsverfassung, wie sie im Vertrag über die Schaffung einer Währungs-, Wirtschafts- und Sozialunion zwischen der Bundesrepublik Deutschland und der Deutschen Demokratischen Republik (Staatsvertrag) vom 18. Mai 1990 festgeschrieben wurde. Dort heißt es:

„Grundlage der Wirtschaftsunion ist die Soziale Marktwirtschaft als gemeinsame Wirtschaftsordnung beider Vertragsparteien. Sie wird insbesondere bestimmt durch Privateigentum, Leistungswettbewerb, freie Preisbildung und grundsätzlich volle Freizügigkeit von Arbeit, Kapital, Gütern und Dienstleistungen (...).Sie wird insbesondere bestimmt durch eine der Sozialen Marktwirtschaft entsprechende Arbeitsrechtsordnung und ein auf den Prinzipien der Leistungsgerechtigkeit und des sozialen Ausgleichs beruhendes umfassendes System der sozialen Sicherung“ (Staatsvertrag 1990: Art 1).

Obwohl an dieser wie an anderen Stellen des Vertrages eines der wesentlichen Anliegen der Sozialen Marktwirtschaft beschrieben wird, nämlich das Prinzip der wirtschaftlichen Freiheit mit dem Prinzip des sozialen Ausgleichs zu verbinden, erscheint das Konzept der Sozialen Marktwirtschaft in diesen Aussagen eher als Handlungsauftrag und politisches Programm und damit für eine Bestimmung des theoretischen Konzepts der Sozialen Marktwirtschaft eher ungeeignet.

Wendet man sich hingegen den theoretischen Schriften zur Sozialen Marktwirtschaft $\mathrm{zu}$, wird deutlich, dass es sich auch hier nicht, wie man angesichts der politischen Bedeutung des Konzepts vermuten sollte, um ein eindeutig bestimmtes Theoriegebäude handelt, sondern die Beiträge zum Konzept der Sozialen Marktwirtschaft unterschiedlichen Schulen und Denktraditionen entstammen. ${ }^{2} \mathrm{Zu}$ den wohl wichtigsten geistigen Vätern des theoretischen Entwurfs der Sozialen Marktwirtschaft zählen zum Einen die Vertreter der so genannten Freiburger Schule, Franz Böhm und Walter Eucken, die in Anlehnung an die von ihnen begründete Zeitschrift, Ordo - Jahrbuch für die Ordnung von Wirtschaft und Gesellschaft' auch zur Schule des so genannten Ordoliberalismus rechnen (vgl. u. a. Pies 2002: 1). Demgegenüber, obwohl ihnen nahe ste-

2 Eine ähnliche Zuordnung, wie im Folgenden dargestellt, treffen u. a. Schefold (2004: 505) und Starbatty (2002: 251ff.). Thieme fasst die ,Schöpfer ${ }^{`}$ des Konzepts der Sozialen Marktwirtschaft unter dem Begriff ,Ordnungstheoretiker zusammen (vgl. Thieme 1994: 23) und auch Starbatty benennt die ,ordnungspolitischen Schlussfolgerungen' als gemeinsames Merkmal der Theoretiker der Sozialen Marktwirtschaft (vgl. Starbatty 2002: 252). 
hend und oftmals im Kontext der ordnungstheoretischen Überlegungen der Freiburger Schule erwähnt, gehört Friedrich August von Hayek nicht zu den ,Gründervätern` der Sozialen Marktwirtschaft. Stärker als Eucken setzt Hayek auf die Prinzipien eines wirtschaftlichen Liberalismus und entwickelt sich im Laufe der Zeit zu einem der größten Kritiker am Konzept der Sozialen Marktwirtschaft (vgl. Aßländer/Ulrich 2009: 12). Vergessen werden darf dabei nicht, dass von Hayek sich zum Zeitpunkt der ,Entstehung' der Sozialen Marktwirtschaft nur am Rande mit der wirtschaftspolitischen Neuausrichtung der gerade erst im Entstehen begriffenen Bundesrepublik Deutschland auseinandersetzte:

„Eucken geht es um einen radikalen Neuanfang für Deutschlands Nachkriegsordnung, während es für von Hayek vordringlich darauf ankommt, die englische Gesellschaft vor einem totalitären Niedergang zu bewahren. Von Hayek warnt vor einem Weg in die Knechtschaft, während Eucken gewissermaßen einen Weg aus der Knechtschaft sucht" (Pies 2001: 133).

Zwei ebenfalls im Umfeld des Ordoliberalismus anzusiedelnde und an der Herausgabe des Ordo-Jahrbuchs beteiligte, jedoch nicht explizit zur Freiburger Schule rechnende Vordenker einer sozial-marktwirtschaftlichen Wirtschaftskonzeption sind Wilhelm Röpke und Alexander Rüstow, die in diesem Konzept eine Art,Wirtschaftshumanismus' verwirklicht sehen wollen, in dem eine marktwirtschaftliche Grundordnung mit sozialer Chancengerechtigkeit in Einklang gebracht werden soll (vgl. Aßländer/Ulrich 2009: 12f.). Eine Außenseiterrolle nimmt in gewissem Sinne der eigentliche Wortschöpfer des Begriffes ,Soziale Marktwirtschaft', Alfred Müller-Armack, ein, der, ausgehend von religionssoziologischen Fragestellungen, deutlicher als die bereits benan nten Vertreter an den ,geistigen' Grundlagen einer Wirtschaftsordnung interessiert ist und stärker als diese auch die politisch-gesellschaftliche Betrachtungsweise des Wirtschaftens in den Vordergrund seiner Überlegungen rückt (vgl. Aßländer/Ulrich 2009: 13).

Was die Vordenker des Konzepts der Sozialen Marktwirtschaft eint, ist zum Einen ihr Eintreten für eine sozial gerechte Gesellschaftsordnung, deren Gestaltung wesentlich durch die Ordnung der Wirtschaft mitbestimmt sei. Dies ist zum Anderen der Glaube an die Vorzugswürdigkeit marktwirtschaftlicher Strukturen und ökonomischen Wettbewerbs als bestem Verfahren zur Steuerung einer bedarfsgerechten Produktion und eines effizienten Warenverkehrs, wobei es aus Gründen der sozialen Gerechtigkeit stets gelte, den Wettbewerb mittels staatlicher Ordnungspolitik , in die richtigen Bahnen' $\mathrm{zu}$ lenken und so unbillige soziale Härten zu vermeiden. In diesem Sinne stützt sich die Theorie der Sozialen Marktwirtschaft im Wesentlichen auf die grundlegenden Vorstellungen einer Wirtschaftsordnung im Sinne des Ordoliberalismus.

Wesentliches Kennzeichen des Konzepts der Sozialen Marktwirtschaft ist somit das Denken in Ordnungen (vgl. Klump 2001: 28). Hierbei genügt es nicht, lediglich der Wirtschaft ihre Ordnung zu geben, vielmehr geht es darum, die Wirtschaftsordnung in eine Gesamtlebensordnung einzubetten. Mit anderen Worten: Rechtsordnung, soziale Ordnung und Wirtschaftsordnung müssen miteinander in Einklang gebracht werden (vgl. u. a. Eucken 1990: 180-184; Müller-Armack 1948a: 104; Röpke 1979a: 161 f.; Lenel 1989: 26). Entsprechend wird Wirtschaft als System innerhalb des Ordoliberalismus stets von der Gesellschaft her gedacht, der zu dienen sie in der Lage sein 
soll (vgl. Röpke 1979a: 23; Müller-Armack 1976: 89). Der marktlichen Steuerung des Wirtschaftsprozesses selbst kommt in diesem System kein Eigenwert zu, sie besitzt lediglich Instrumentalcharakter, da sie am besten dazu geeignet ist, die moralischen Ziele der Gemeinschaft - i. e. Reduktion von Knappheit bei gleichzeitig weitestgehender Ermöglichung von Freiheit und sozialer Gerechtigkeit - zu gewährleisten (vgl. u. a. Eucken 1990: 317; Röpke 1994: 331f.; Müller-Armack 1948b: 86). Dabei ist den meta-ökonomischen Zielen, wie Freiheit und Gerechtigkeit, im Zweifel Vorrang vor den ökonomischen Zielen, insbesondere dem Wirtschaftswachstum, einzuräumen (vgl. u. a. Müller-Armack 1962: 20; Dreier 1990: 108). Norman P. Barry bezeichnet dies als die Verflechtung der ökonomischen mit den weiteren sozialen Imperativen innerhalb der Konzeption der Sozialen Marktwirtschaft und bringt dies in Anlehnung an Alexander Rüstow auf den Punkt (Barry 1989: 108):

„The social market economy must be the servant of humanity and of transeconomic values. All social, ethical, cultural and human values are more important than the economy, yet the economy must prepare the ground for their fullest development. For this reason the economy must not take on forms which are incompatible with these trans-economic values."

Das Konzept der Sozialen Marktwirtschaft ist daher als ein Gesellschaftsentwurf zu verstehen, der die Elemente Freiheit, soziale Gerechtigkeit und Eigenverantwortung miteinander in Einklang bringen möchte. Tragend für dieses Konzept ist der Gedanke, dass die wirtschaftliche Ordnung zum Einen auf der gesellschaftlichen Ordnung, ihren Moralvorstellungen, Werthaltungen und Zielsetzungen aufruhe - Röpke (1979c: 85) spricht von einem der Wirtschaft „widergelagerten Gesellschaftssystem“ -, und zum Anderen durch den Staat und seine Organe in die gesellschaftspolitisch erwünschten Bahnen gelenkt werden müsse (vgl. Aßländer/Ulrich 2009: 9).

Zu diesem Zweck entwirft Eucken eine Wirtschaftsordnung, die mit der (erwünschten) gesellschaftlichen Ordnung kompatibel ist und so die Umsetzung vorgängiger, zentraler gesellschaftlicher Werte wie Freiheit oder soziale Gerechtigkeit unterstützt. Ziel der,Wettbewerbsordnung' ist die staatliche Ordnung der Märkte, die Aufrechterhaltung eines leistungsbezogenen Wettbewerbs und die Garantie der Planungsautonomie der Haushalte und Unternehmen (vgl. Eucken 1949: 22f.; Eucken 1990: 245f.). Im Wesentlichen basiert die Wettbewerbsordnung dabei auf der Bestimmung konstituierender Prinzipien, deren Aufgabe es ist, den Ordnungsrahmen des Wettbewerbs festzulegen, und regulierender Prinzipien, die negativen Entwicklungen innerhalb der Wirtschaft möglichst automatisch entgegenwirken und den Wettbewerb funktionsfähig erhalten sollen. Während die erstgenannten Prinzipien gleichsam die staatlich garantierten institutionellen Voraussetzungen des Wirtschaftens darstellen, ist es Aufgabe der letztgenannten, das Marktsystem vor seinen eigenen selbst zerstörerischen Kräften zu schützen (vgl. Barry 1989: 114f.).

Angesichts der verheerenden Krisen von 1919 und 1929 gilt das besondere Augenmerk im Konzept der Wettbewerbsordnung der Herstellung funktionsfähiger Preismechanismen sowie der Währungsstabilität, die Eucken zu den wichtigsten konstituierenden Prinzipien rechnet: 
„Alle Bemühungen eine Wettbewerbsordnung zu verwirklichen, sind umsonst, solange eine gewisse Stabilität des Geldwertes nicht gesichert ist. Die Währungspolitik besitzt daher für die Wettbewerbsordnung ein Primat“" (Eucken 1990: 256).

Insbesondere warnen die Väter der Sozialen Marktwirtschaft vor den Gefahren der währungspolitischen Destabilisierung durch die derivative Geldschöpfung der Kreditbanken und plädieren nicht nur für eine aktive Geldmengensteuerung durch eine unabhängige Notenbank und eine strikte Bankenaufsicht sondern zugleich auch für einen Umbau des bisherigen Bankensystems, der es den Banken verunmöglichen soll, eine eigenständige Geldpolitik zu betreiben (vgl. Eucken 1990: 258ff.). Nur eine stabile Wirtschafts- und Währungsordnung ist in der Lage, Krisen zu verhindern und die internationale Wirtschaft zu stabilisieren:

„Der Fall der Weltwirtschaft beweist also in besonders eindrucksvoller Weise, dass die internationale Ordnung die nationale Ordnung voraussetzt. Dasselbe gilt für die Ordnung des internationalen Zahlungsverkehrs, ohne die eine wirkliche Weltwirtschaft nicht zu denken ist" (Röpke 1979d: 29). ${ }^{3}$

\section{Ursachenanalyse - Die aktuelle Wirtschaftskrise vor dem Hintergrund der Konzeption der Sozialen Marktwirtschaft}

Das Konzept der Sozialen Marktwirtschaft versteht sich in seinen Ursprüngen als eine nationalstaatliche Wirtschafts- und Gesellschaftsordnung, was sich nicht zuletzt aus der Idee der staatlich garantierten Wettbewerbsordnung ergibt. Jenseits der Klage über die mangelnden Finanzierungsmöglichkeiten eines ,Sozialstaates' (vgl. u. a. Sesselmeier 2007: 110-116; Igel/Wille 2007: 250-264), die sich in erster Linie an der spezifischen Ausgestaltungsform des bundesdeutschen Sozialversicherungswesens entzündet, bemängeln Kritiker daher vor allem die vermeintliche Untauglichkeit der Sozialen Marktwirtschaft, den neuen Herausforderungen der Globalisierung zu begegnen (vgl. Münchau 2006). Zahlreiche Reformen der letzten Jahre sind daher der Anpassung des Konzepts der Sozialen Marktwirtschaft an die Herausforderungen einer globalen Wirtschaft geschuldet. Dies ist durchaus mit den Intentionen der Gründerväter vereinbar, die die Soziale Marktwirtschaft als eine „dynamische Ordnungskonzeption“ (Thieme 1994: 23) verstanden wissen wollten, die entsprechend der jeweils aktuellen gesellschaftlichen Entwicklungen fortgeschrieben werden müsse (vgl. Eucken 1949: 29; Müller-Armack 1948a: 141).

„Der Spannungs- und Konfliktzustand unserer Gesellschaft unterliegt selbstverständlich dem geschichtlichen Wechsel und verlangt, dass die jeweils strategischen Formeln dieses irenischen Ausgleichs immer wieder neu gesucht werden müssen, um ihrer Aufgabe gewachsen zu sein. Soziale Marktwirtschaft ist so eine Strategie im gesellschaftlichen Raum; ob sie gelingt und ihr Ziel erreicht, wird nie exakt entschieden werden können, sondern bestätigt sich nur im dauernden Pro-

3 Allerdings gehen auch hier die Meinungen darüber, wie sich Geldwert und intemationaler Zahlungsverkehr stabilisieren ließen, bei den Theoretikern der Sozialen Marktwirtschaft auseinander. Während Röpke beispielsweise den Goldstandard favorisiert (Röpke 1979d: 29f.; Röpke 1994: 150f.), spekuliert Eucken über ein System der Waren-Reserve-Währung (Eucken 1990: 261f.) 
zess der Lösung jener internen Konflikte unserer Gesellschaft, die wir als Realität hinzunehmen haben“ (Müller-Armack 1962: 13f.).

Entsprechend erfolgten im Laufe der Zeit mehrere „Kurskorrekturen“ der Sozialen Marktwirtschaft. So kam es mit der Rezession von 1967 zu einer ersten Umorientierung der deutschen Wirtschaftspolitik, die sich nun zunehmend an einem keynesianischen Konzept der wirtschaftlichen Globalsteuerung auszurichten begann (vgl. Thieme 1994: 98; Zinn 1992: 51), was von einigen Kritikern, nicht ganz zu unrecht, ${ }^{4}$ als Abkehr vom Konzept der Sozialen Marktwirtschaft kritisiert wurde (vgl. Lenel 1971; Lenel 1989: 31f.). Mitte der 1980er Jahre kam es zu einer erneuten Kurskorrektur hin zu einer unternehmerfreundlichen und vorrangig angebotsorientierten Wirtschaftspolitik, wobei die bis dahin stark betonte gesellschaftspolitische Dimension der Sozialen Marktwirtschaft in den Hintergrund trat (vgl. Hauser 2007: $91 \mathrm{f}$.); die Aufsicht des Staates über die Entwicklung der Märkte wurde schrittweise zugunsten einer Dynamisierung der Wirtschaft zurückgefahren (vgl. ausführlich Hübinger 2001).

Obwohl sich die Soziale Marktwirtschaft als gestaltungsoffenes System versteht, bleibt die Frage, ob die wirtschafts- und gesellschaftspolitischen Anpassungen insbesondere seit den 1980er Jahren tatsächlich mit jenen Vorstellungen einer sozialen Marktwirtschaft vereinbar sind, wie sie von ihren Theoretikern formuliert wurden. Da sich das Konzept der Sozialen Marktwirtschaft als gesamtgesellschaftlicher Entwurf versteht, soll im Folgenden vor dem Hintergrund der sozial-marktwirtschaftlichen Ordnungskonzeption der Frage nach den Fehlentwicklungen in Politik, Wirtschaft und Gesellschaft nachgegangen werden, die möglicher Weise mit ursächlich für die aktuelle Wirtschaftskrise sind.

\subsection{Fehlsteuerung durch angebotsorientierte Politik}

Innerhalb der Politik lassen sich mindestens zwei Entwicklungen aufzeigen, denen für den Verlauf der Krise eine besondere Bedeutung zugemessen werden muss. Es ist dies zum Einen die im Zuge der Umorientierung nach 1980 erfolgte Welle an Deregulierungen der Märkte und es ist dies zum Zweiten das damit in gewisser Weise einhergehende Versäumnis einer effizienten Finanzmarktkontrolle.

Die Neuausrichtung der deutschen Wirtschaftspolitik der Nachwendezeit war bestimmt von einer Politikrhetorik, die vor allem den Wettbewerb der Ordnungssysteme und die vermeintlich mangelnde Zukunftsorientierung des Wirtschaftsstandorts Deutschland betonte (vgl. Schmidt 2008). Im Zuge der Globalisierung, so das erste Argument, geriete das sozial-marktwirtschaftliche Konzept selbst unter ,Wettbewerbsdruck'. Es bedürfe daher weit reichender ordnungspolitischer Reformen, um die bundesdeutsche Wirtschaftsordnung konkurrenzfähig auszugestalten (vgl. Schmidt 2009: 196; Blum 2009: 190f.). Besondere Gefahren drohen der Sozialen Marktwirt-

4 So erweist sich insbesondere Wilhelm Röpke als einer der schärfsten Gegner des Keynesianismus: „Wenn es keinen Keynes, besser gesagt keinen Verfasser des Buches ,The General Theory of Employment, Interest and Money' gegeben hätte, so wäre die Wissenschaft der Nationalökonomie möglicherweise um manches ärmer, aber die Völker wären um so viel reicher, als die Gesundheit ihrer Wirtschaft und Währung weniger durch Inflation gefährdet wäre“ (Röpke 1979a: 275). 
schaft dabei durch einen ruinösen Steuerwettbewerb, der zu einer Unterfinanzierung bei der Erstellung öffentlicher Güter führe, zugleich das soziale Sicherungssystem bedrohe und schließlich in einen ruinösen Deregulierungswettbewerb münden würde (vgl. Schmidt 2009: 201). In der Folge kam es daher zu weit reichenden Liberalisierungen beispielsweise im Gesundheitswesen und zu einem Rückbau der staatlichen Sicherungssysteme, insbesondere im Bereich der Altersvorsorge.

Der zweite Vorwurf betraf vor allem die angeblich mangelnde Wachstumsorientierung der bundesrepublikanischen Wirtschaftsordnung. So baue die Kreditvergabepraxis der meisten Geschäftsbanken zu sehr auf Sicherheiten und die Unternehmen seien zu wenig am Kapitalmarkt orientiert (vgl. Habisch 2001: 197ff.; Münchau 2006: 112ff.; Hengsbach 2009: 303). International konkurrenzfähige Wirtschaftssysteme zeichneten sich demgegenüber durch Wachstumsorientierung, flexible Arbeitsmärkte, leichten Zugang zu Risikokapital und innovative Finanzmärkte aus. Entsprechend zielten die Reformbestrebungen der Bundesregierungen auf eine Flexibilisierung des Arbeitsmarktes und - nicht zuletzt auch im Zuge der europäischen Vereinheitlichung - auf eine Deregulierung des Finanzmarktes.

Die Vorgaben der angebotsorientierten Wirtschaftspolitik ließen staatliche Rahmenvorgaben im Sinne der Sozialen Marktwirtschaft zunehmend unpopulärer erscheinen. Schrittweise zog sich die Politik aus ihrer Verantwortung für die Steuerung des Wirtschaftsgeschehens und für die Regulierung der Märkte zurück. War es im Konzept der Sozialen Marktwirtschaft zentrale Aufgabe der Wirtschaftspolitik, die Märkte zu beaufsichtigen und Fehlentwicklungen nötigenfalls durch staatliche Eingriffe entgegenzuwirken (vgl. u. a. Kloten 1989: 69ff.; Möschel 1989: 151 ff.), wurde dies nun mit dem Hinweis auf die effiziente Selbstregulierung der Märkte weitgehend abgelehnt.

Mit dem Investmentgesetz von 2004 reformierte die Bundesregierung das bis dahin gültige Gesetz über Kapitalanlagegesellschaften und das Auslandsinvestment-Gesetz mit dem Ziel, die Wettbewerbsfähigkeit des Finanzplatzes Deutschland zu erhöhen. Sie schuf damit zugleich die Grundlage für die Zulassung von Hedgefonds, die zumindest in Form so genannter Dachfonds öffentlich vertrieben werden können, erweiterte die Anlagemöglichkeiten der Fonds in Derivaten und lockerte die Vorschriften für die Eigenkapitalausstattung von Finanzdienstleistern (vgl. u. a. Hengsbach 2009: 304f.). Dies führte jedoch nicht nur zu einer Flexibilisierung des Finanzmarktes sondern erhöhte im Zweifel auch das Risikopotenzial der auf den Märkten angebotenen Finanzprodukte.

Als problematisch erwies sich, dass es die nun vor allem an wirtschaftlichem Wachstum orientierten Verantwortlichen der Politik damit verabsäumten, jenes zentrale konstituierende Prinzip im Auge zu behalten, das Eucken als oberstes Prinzip der Wettbewerbsordnung bezeichnet, da er in einer preisstabilen Währung die unabdingbare Voraussetzung für die Aufrechterhaltung eines funktionsfähigen Wettbewerbs und die Grundlage für wirtschaftliche Prosperität sah. Um eine unkontrollierte Geldpolitik der Geschäftsbanken zu verhindern, hatte Eucken einen Umbau des Bankwesens und eine strikte Bankenaufsicht gefordert (vgl. Eucken 1990: 258-262). In ähnlicher Weise hatte vor ihm bereits Wilhelm Röpke 1937 vor den desaströsen Folgen einer unkontrollierten derivativen Geldschöpfung für eine stabile Wirtschaftsordnung gewarnt: 


\begin{abstract}
„Eine Bank ist eben nicht länger ein Geschäftunternehmen wie jedes andere; es ist keine bloße Geldgarderobe (...), sondern ein Unternehmen dessen Geschäfte einen weitreichenden Einfluss auf die Geldzirkulation und damit auf den gesamten Wirtschaftsprozess ausüben, ein Unternehmen daher auch, das unkontrolliert sich selbst zu überlassen sogar dem hartgesottensten Liberalen kaum jemals in den Sinn gekommen ist" (Röpke 1994: 133).
\end{abstract}

Allerdings bezogen sich Röpkes und Euckens Bedenken vor allem auf die Möglichkeit der derivativen Geldschöpfung durch Kreditvergabe. Nicht in den Blick kam dabei die Möglichkeit, die Geldmenge durch eine Überbewertung von Forderungsbeständen oder gar durch den Handel mit zu Wertpapieren umgewandelten Forderungen ausweiten zu können, was eine Geldschöpfung auch jenseits des klassischen Kreditgeschäftes der Geschäftsbanken ermöglichte (vgl. Münchau 2008: 124f.).

Es lässt sich zeigen, dass die Möglichkeit der Finanzinstitute, durch den Handel mit zu strukturierten Finanzprodukten umgewandelten Hypotheken stetig neue Liquidität für weitere Kreditvergaben zu generieren, eine der zentralen Ursachen der derzeitigen Wirtschaftskrise darstellt. Als dramatisch erwies sich nun, dass aufgrund der im Zuge des Investmentgesetzes gelockerten Eigenkapitalvorschriften und angesichts ihrer überhöht ausgewiesenen Vermögensbestände den Banken nun die Überschuldung drohte (vgl. u. a. Sinn 2010a: 117-122; 193-203). Um einen Zusammenbruch des Bankensystems zu vermeiden, sah sich die Bundesregierung gezwungen, die Banken mit neuem Geld zu versorgen und zugleich die Bilanzierungsregeln zu ändern, d. h. den Banken eine Bilanzierung ihrer, Vermögenswerte‘ zu anderen als den Marktpreisen zu gestatten, um so einer Überschuldung der Banken und damit weiteren Konkursen im Bankensektor vorzubeugen (vgl. Münchau 2008: 66f.).

Mit aller gebotenen Vorsicht muss konstatiert werden, dass der zunehmende Glaube der Regierungsverantwortlichen an die Selbststeuerung der Märkte, die Liberalisierung der Märkte und der mangelnde Wille der Regierungsverantwortlichen, ihre Regulierungskompetenz innerhalb des sozial-marktwirtschaftlichen Systems emst zu nehmen, eine der wohl wichtigsten Ursachen für die Wirtschaftskrise darstellten (vgl. Sinn 2010a: 367). So bemerkt Hans-Werner Sinn völlig zu Recht:
„(d)ass die Regulierung des Bankensystems nicht funktioniert hat und dass die Vorstellung, das Bankensystem könne sich selber regulieren, abwegig ist. Die Risiken, die die Banken eingehen, verursachen solche massiven externen Effekte auf den Rest der Weltwirtschaft, dass es einer international harmonisierten Regu- lierung bedarf. Das haben inzwischen alle eingesehen" (Sinn 2010b).

Zwar muss mit Bernd Hübinger (2001) festgehalten werden, dass die Abkehr vom Gedanken einer keynesianischen Globalsteuerung durchaus einer Rückbesinnung auf die Prinzipien der Sozialen Marktwirtschaft entsprach. Allerdings führten die seitens der Regierungsverantwortlichen eingeleiteten Maßnahmen in jenen Punkten zu weit, in denen sie das Prinzip der Wettbewerbsordnung zugunsten einer neoliberalen Wachstumspolitik verletzten. So wurde weder den mit dem Aufkommen neuer Finanzprodukte geschaffenen Möglichkeiten der Geschäftsbanken zur derivativen Geldschöpfung Beachtung geschenkt, noch eine effektive Kontrolle über das Finanz- und Bankensystem ausgeübt, wie dies als zentrale Forderung von den Theoretikern der Sozialen Marktwirtschaft erhoben wird. Allzu sehr vertrauten die Regierungsverant- 
wortlichen darauf, durch den Abbau staatlicher Beschränkungen neue Wettbewerbskräfte freizusetzen und so Wirtschaftswachstum zu fördern und Arbeitslosigkeit zu bekämpfen. Damit wurden der Instrumentalcharakter der Wirtschaft und ihre notwendige Einbettung in ein gesamtgesellschaftliches Zielsystem aus den Augen verloren:

„Wettbewerb allein kann die Energiefreisetzung zwar fördern, aber da Wettbewerb ein ,seltsamer Attraktor' ist, der keine Richtung hat und auch zu schlechteren Produkten und wirtschaftsschädlichen Verhaltensweisen führen und somit auch zerstörerisch für das System wirken kann, reicht Wettbewerb als einzige Koordinierungsregel nicht aus“" (Hauser 2007: 83).

\subsection{Fehlsteuerung durch falsche Anreize für die Wirtschaft}

Zu den in der medialen Diskussion wohl am häufigsten genannten Ursachen der Wirtschaftskrise zählen die fehlerhaften Anreizstrukturen innerhalb der Wirtschaft. Dies betrifft zum Einen die staatlichen Anreizstrukturen, die sich auf die jeweilige Unternehmenspolitik der Finanzinstitute auswirken, und zum Anderen die Anreizstrukturen der Unternehmen, die letztlich das Mitarbeiter- und Managerverhalten bestimmen.

Mit „Basel I` und ,Basel II` schuf der Baseler Ausschuss für Bankenaufsicht ein internationales Regelwerk für die Eigenkapitalausstattung von Geschäftsbanken, das für alle Banken der Europäischen Union verbindlich ist. Ziel war es, eine angemessene Eigenkapitalausstattung der Banken sicher zu stellen. So schrieb „Basel I‘ eine Eigenkapitalquote der Banken von acht Prozent vor, wobei das hierzu in Bezug gesetzte Kreditvolumen nach einheitlichen Kriterien gewichtet wurde (vgl. Münchau 2008: 78). Mit ,Basel II' wurden die starren Bewertungsvorschriften zum Teil dahingehend ,gelockert $^{6}$, dass die Bewertung des Kreditrisikos nun den Banken selbst überlassen wurde, die die ausstehenden Kreditforderungen je nach Bonität des Kreditnehmers zwischen null und einhundertfünfzig Prozent gewichten konnten (vgl. Bloss et al. 2009: 52f.; Sinn 2010a: 193-197). Um die Bindung von Eigenkapital durch die risikogewichteten Aktiva zu verhindern, gingen Banken dazu über, 'Zweckgesellschaften' zu gründen, auf die die Risken übertragen wurden. Dies erlaubte es den Banken, ihr Kreditvolumen bei gegebener Eigenkapitalausstattung stetig auszuweiten, ohne dass die nun ausgelagerten Kreditrisiken durch das Eigenkapital der Banken gedeckt waren. Letztlich führte so die Regelung, die ursprünglich zur Sicherung einer angemessenen Eigenkapitalquote bei den Geschäftsbanken gedacht war, zum genauen Gegenteil dessen, was eigentlich beabsichtigt war.

Auch innerhalb der Finanzunternehmen lässt sich in mehrfacher Hinsicht ein System fehlerhafter Anreizstrukturen konstatieren. Dies betrifft zum Einen die fragwürdigen Erfolgsbeteiligungen amerikanischer Hypothekenverkäufer, die, nach Anzahl der Hypothekenverkäufe bewertet, die Bonitätsprüfungen ihrer Kunden zunehmend laxer handhabten. Dies betrifft aber auch die vor allem an einer hohen Eigenkapital rendite interessierten deutschen Geschäftsbanken, die über entsprechende Gratifikationssy steme gezielt Anreize setzten, die Eigenkapitalvorschriften zu umgehen. Die Liste fehlerhafter Anreizstrukturen ließe sich an dieser Stelle beliebig verlängern. 
Analysiert man diese Anreizstrukturen jedoch genauer, zeigt sich, dass es weit weniger fehlerhafte Anreize waren, die das Fehlverhalten der einzelnen Marktakteure beeinflussten, sondern deren Wunsch, sich durch Umgehung der bestehenden Regeln Vorteile zu verschaffen. So waren es nicht ,Basel I` oder ,Basel II‘ die zur Gründung von Zweckgesellschaften und zur Auslagerung von Krediten zwangen, sondern der Wunsch der Beteiligten, durch Umgehung der Beschränkungen die eigenen geschäftlichen Möglichkeiten zu erweitern. Auch die erfolgsabhängige Entlohnung amerikanischer Hypothekenverkäufer beinhaltete keine Aufforderung, die Regeln für die Bonitätsprüfung bei der Kreditvergabe eigenmächtig zu lockern. So berechtigt daher der Ruf nach einer strikteren Vorschrift zur Eigenkapitalausstattung von Banken im Rahmen einer ,Basel III‘ Regulierung auf der einen Seite auch sein mag (vgl. Sinn 2010a: 379-383), so unterschätzt er doch den Willen der Beteiligten, auch diese neuen Regulierungen im Interesse der Eigennutzmaximierung zu umgehen.

Damit ist ein Kernproblem der Wirtschaftsordnung offensichtlich: Das Funktionieren der Märkte hängt nicht nur von der Ordnung der Märkte ab, sondern auch vom Willen der Marktteilnehmer, diese Regeln als verbindlich zu befolgen. Lin-Hi und Suchanek (2009: 24f.) sprechen in diesem Zusammenhang von ,Spielverständnis' der Akteure und meinen damit eben jene freiwillige Regelbindung, die die Umsetzung von allgemeinverbindlichen Spielregeln überhaupt erst ermöglicht.

Dass ein regelkonformes Verhalten der Marktakteure Voraussetzung einer Wettbewerbsordnung ist, war dabei bereits für die Väter der Sozialen Marktwirtschaft offensichtlich (vgl. Kloten 1989: 70f.). Obwohl bei der Begründung seiner Wettbewerbsordnung vor allem um eine schlüssige ökonomische Argumentation bemüht, weiß Walter Eucken, dass die Einhaltung der Wettbewerbsregeln nicht zuletzt auch von der Moral der Wirtschaftsakteure und deren Einsicht in die Richtigkeit der Regeln abhängt. Um der Wirtschaft eine funktionsfähige und menschenwürdige Ordnung zu geben (vgl. Eucken 1989: 240) und diese auf Dauer zu stellen und langfristig funktionsfähig zu erhalten, bedarf es zwar grundlegender ordnungspolitischer Maßnahmen - staatspolitische Grundsätze, konstituierende und regulierende Prinzipien der Wettbewerbsordnung -, wie Ingo Pies in seiner Analyse des Euckenschen Ansatzes zu Recht ausführt (Pies 201: 62f.; 2002: 18f.). Doch übersieht Pies dabei, dass auch Wettbewerbsregeln von den moralischen Regelbindungen derjenigen abhängen, die sie befolgen sollen. Eucken rekurriert daher auch auf die Moral der Wirtschaftsakteure und fordert, dass keiner der Wirtschaftsakteure sich ausschließlich egoistisch verhalten dürfe, da hierdurch die Wettbewerbsordnung gefährdet würde (vgl. Eucken 1990: 354). Wesentlich deutlicher noch als Eucken formuliert Wilhelm Röpke diese Einsicht und zeigt sich hinsichtlich der Steuerungsfähigkeit einer Wettbewerbsordnung, die nicht zugleich auf gesellschaftlich anerkannten und allgemein befolgten moralischen Prinzipien aufruht, noch erheblich pessimistischer:

„Menschen, die auf dem Markte sich miteinander im Wettbewerb messen und dort auf ihren Vorteil ausgehen, müssen um so stärker durch die sozialen und moralischen Bande der Gemeinschaft verbunden sein, andernfalls auch der Wettbewerb aufs schwerste entartet“ (Röpke 1979a: 146).

Und obwohl Röpke davon überzeugt ist, dass der „Rechenmeister Markt“ (Röpke 1979a: 59) für eine Wirtschaftsordnung unverzichtbar sei, und der Markt vor Preis 
verzerrenden, staatlichen Eingriffen unbedingt geschützt werden müsse, bleibt er hinsichtlich der ,moralischen Folgen“ des Wettbewerbs skeptisch. Für ihn sind Selbstdisziplin, Gerechtigkeitssinn, Ehrlichkeit, Fairness und Maßhalten sittliche Normen, ohne die eine Wettbewerbswirtschaft auf Dauer nicht funktionsfähig erhalten werden kann (vgl. Röpke 1979a: 186; Röpke 1979b: 214). Damit wird das Funktionieren einer Wettbewerbsordnung auch zur Frage der Moral der Wettbewerbsakteure. Zwar lässt sich hinsichtlich der Möglichkeit zu ihrer Umgehung durchaus zwischen ,besseren“ und ,schlechteren' Regeln unterscheiden - insofern ist Hans-Werner Sinns Forderung einer glaubhaften Regulierung zuzustimmen (vgl. 2010a: 384-387); gleiches gilt wohl auch für die durch die Regeln gesetzten Anreizstrukturen. Dennoch gilt es jenseits der Frage nach den ,richtigen' oder ,falschen' Anreizstrukturen auch auf die Mitverantwortung der Akteure des Finanzmarktes zu verweisen, die sich von falschen Anreizen zur wissentlichen Regelumgehung haben verleiten lassen. Hier alleine darauf zu bauen, dass sich der ordnungstheoretische Ansatz problemfrei von einem ,Denken in Ordnungen' in ein ,Denken in Anreizen“ überführen lasse, und zu hoffen, dass die ,richtigen'staatlichen Anreizsysteme wie von selbst das moralische Wohlverhalten der Wirtschaftsakteure sicherzustellen in der Lage seien (vgl. Pies 2002: 31, ähnlich Sinn 2010a: 14) scheint angesichts der Analysen der aktuellen Wirtschafts- und Finanzmarktkrise und vor dem Hintergrund der Warnungen Euckens und Röpkes als mindestens problematisch. Zwar sieht Hans-Werner Sinn die Krise durchaus als „das Ergebnis der Unfähigkeit der internationalen Staatengemeinschaft, ein einheitliches Regulierungssystem für Banken und andere Finanzinstitute zu schaffen" - nennt als deren Ziel jedoch, „den Eigennutz der Akteure so (zu) kanalisier(en), dass er sich segensreich und produktiv entfalten kann, wie man es von einer Marktwirtschaft erwartet" (Sinn 2010a: 14).

Jenseits der Frage, ob es tatsächlich Euckens Programm gewesen sei, „die Interessengruppen mit Hilfe intellektueller Orientierungsleistungen in die Lage zu versetzen, ihre Interessen wirksam(er) zur Geltung zu bringen“" (Pies 2001: 24), muss zumindest hervorgehoben werden, dass die Verfolgung eigener Interessen im Sinne Euckens nicht als eine rein auf egoistischen Vorteilüberlegungen basierende Interessenverfolgung missverstanden werden darf. Für ihn bleibt der Markt reines Koordinierungsinstrument, das den Einzelnen bei der Verfolgung seiner Zwecke (ob egoistisch oder altruistisch) zur möglichst effizienten Mittelwahl zwingt und gerade hierdurch die Vorzugswürdigkeit gegenüber anderen (wirtschaftlichen) Koordinierungsinstrumenten unter Beweis stellt.

„Egoismus und Altruismus bestimmen die Zwecke, auf welche die Wirtschaftspläne ausgerichtet werden; durch Handeln nach dem wirtschaftlichen Prinzip wird die Auswahl der Mittel bestimmt, um die Zwecke zu erreichen. ,Egoismus' und ,wirtschaftliches Prinzip“ befinden sich also gleichsam auf unterschiedlichen Ebenen“ (Eucken 1990: 353).

Festzuhalten bleibt, dass das Bankengeschäft vor allem von institutionellen Anlegern bestimmt ist, die mehrheitlich an Spekulationsgewinnen auf den Kapitalmärkten interessiert sind. Entsprechend hat sich der Mythos innerhalb der Wirtschaft verbreitet, dass es vordringlichste Aufgabe des Managements sei, den Wert dieser Kapitalanlagen (shareholder value) zu steigern. Hieraus ergibt sich die Tendenz, dass sich die aus- 
schließlich an Aktienratings und Börsenkursen orientierte und in gesteigertem Maße auf mathematischen Modellen und Prognosen basierende monetäre Sphäre der Wirtschaft zunehmend von der realwirtschaftlichen Entwicklung löst (vgl. Hengsbach 2009: 301). Insbesondere in der am angelsächsischen Vorbild orientierten Schulökonomie lässt sich hier eine Entwicklung feststellen, die den Vorstellungen der Väter der Sozialen Marktwirtschaft vom Zweck des Wirtschaftens als „servant of humanity and of trans-economic values“" (Barry 1989: 108) diametral entgegengesetzt zu sein scheint. Dabei sollten sich insbesondere die Ökonomen vor Augen halten, so Röpke (1979a: 368),

„(...) dass die Nationalökonomie selbstverständlich keine Naturwissenschaft (...), sondern eine Geisteswissenschaft ist und es als eine solche ,moral science" mit dem Menschen als einem geistig-moralischen Wesen zu tun hat.“

\subsection{Fehlsteuerung durch überzogene Erwartungshaltungen in der Gesellschaft}

Eine der wohl populärsten Ursachenbeschreibungen der Finanz- und Wirtschaftskrise ist der Verweis auf die Gier der Manager, die vor allem an hohen Bonuszahlungen und zusätzlichen Gratifikationen interessiert seien. Jedoch würde man es sich zu einfach machen, wollte man die Schuldigen der Finanz- und Wirtschaftskrise nur in den Reihen scheinbar gieriger Manager und Vorstände suchen. ,Avaritia' ist keineswegs ausschließliches ,Privileg' der Manager und zählt wohl nicht ohne Grund zu den Todsünden innerhalb der katholischen Kirche. Letztlich beschreibt das Mehr-Haben-Wollen eine der Grundkonstanten des menschlichen Daseins und wird von Adam Smith nicht ganz zu Unrecht als wesentliches Motiv für wirtschaftliche Betätigung beschrieben.

Allerdings scheint sich der Wunsch nach höherem Einkommen und besseren Leben sbedingungen zunehmend mehr auf den Wunsch nach arbeitslosen Einkommen zu beziehen. Wie anders wäre es zu erklären, dass zehntausende deutsche Kleinanleger mehrere hundert Millionen Euro auf Konten der isländischen Kaupthing Bank anlegten, die mit weit über dem Marktdurchschnitt liegenden Zinsen gelockt hatte (vgl. Baltzer/Ruhkamp 2009). Galt den konservativen Deutschen bis vor wenigen Jahrzehnten der sprichwörtliche Lottogewinn noch als das Ideal des ,leistungslosen` Einkommens, hat sich diese Sichtweise mit den Aktienbooms und den damit verbundenen Spekulationsgewinnen der letzten beiden Jahrzehnte verändert. Peter Sloterdijk plädiert daher dafür, anstatt von der Gier der Manager von einer Schatzgräbermentalität der breiten Bevölkerung zu sprechen:
„Seit dem Beginn der Neuzeit hat sich in den Menschen Europas und Amerikas das Märchenmotiv vom leistungslosen Einkommen mit archetypischer Gewalt festgesetzt. Von unserer psychischen und kulturellen Struktur her sind wir Schatz- sucher, die den Schatz nicht mehr im Jenseits, sondern auf der Erde vermuten. Wenn es um Reichtum geht, neigen wir zum Wunderglauben - daneben sind mittelalterliche Menschen pure Rationalisten. Unzählige meinen allen Ernstes, das Leben sei ihnen einen Schatzfund schuldig“" (Sloterdijk 2009).

Zu einer ähnlichen Einschätzung kommt auch der Soziologe Christoph Deutschmann, wenn er feststellt, dass es nicht nur die ,Superreichen' sondern insbesondere 
auch die Anleger aus der Mittelschicht seien, die unverantwortlich handeln. Gesellschaftlich angehalten, sich in Geldangelegenheiten möglichst rational bzw. ökonomisch sinnvoll zu verhalten, sucht diese Gruppe möglichst rentable Anlagemöglichkeiten. Den Hintergrund hierfür bildet nicht zuletzt das Bestreben, die eigene, durch den Besitz von Geld begründete, gesellschaftliche Position zu sichern.

„Individuelle Anleger pflegen sich über das Problem der kollektiven Folgen des eigenen Handelns keine großen Gedanken zu machen. Weit verbreitet ist die Vorstellung, man habe so etwas wie ein ,Naturrecht' auf Rendite. (...) Woher der Gewinn eigentlich kommt: darüber denkt man nicht genauer nach. (...) Der Gewinn scheint aus dem Portfolio zu fließen, wie der Strom aus der Steckdose“" (Deutschmann 2008: 509)

In einer zunehmend rein an ökonomischen Erfolgsgrößen orientierten Wirtschaft scheint sich die Frage nach dem gesellschaftlichen Zweck des Wirtschaftens nicht mehr zu stellen; die dem Wirtschaften vorgängigen Werte, wie Subsidiarität und Solidarität als grundlegende Voraussetzungen einer sozial-marktwirtschaftlichen Wirtschaftsordnung (Röpke 1979c: 85) werden, so hat es den Anschein, der modernen Form der ,Avaritia‘ als Gier auf stetig steigende Aktienkurse und immer größerer Kursgewinne geopfert.

„Heute hingegen hat sich ein Fortuna-Kult durchgesetzt, mit dem die Göttin des Zufalls gefeiert wird. Was aber ist ungerechter als der Zufall? Im Grunde genommen leben wir seit dem 19. Jahrhundert in einer neofatalistischen Religion, in der man eine launenhafte Göttin, die Freundin der Sieger, anbetet. Sie ist die Göttin der Stadien, die Göttin der Börsen und die Göttin der erotischen Duelle, sie ist immer zur Stelle, wo es Sieger und Verlierer gibt. Ihre auffälligste Eigenschaft ist, dass sie nie sagt, warum sie den einen bevorzugt und den anderen ignoriert. Begründungen sind nicht ihre Stärke“"(Sloterdijk 2009).

Für die Väter der Sozialen Marktwirtschaft und insbesondere Walter Eucken galt Wettbewerb als Mittel zur Verwirklichung von Freiheit und Sozialer Gerechtigkeit (vgl. Pies 2001: 59). Wird marktlicher Wettbewerb jedoch zum reinen Selbstzweck und nicht mehr als Instrument für eine möglichst optimale Güter- und Faktorallokation betrachtet, wird Gewinn(en) und egoistisches Erfolgsstreben zu Lasten anderer zum Grundmotiv ,wirtschaftlicher' Tätigkeit, und geht die einzelwirtschaftliche Betätigung im Zweifel zu Lasten der Allgemeinheit, verliert die Wirtschaft ihren Bezug zur Gesellschaft.

„Wenn Freiheit dabei zur bloßen Marktfreiheit wird, Gerechtigkeit im Sinne des Äquivalenzprinzips als Tauschgerechtigkeit verengt und Ordnung als umfassender Zielwert politischen Handelns dem Prinzip der Marktkonformität unterstellt wird, dann sind unter der Hand die gesellschaftspolitischen Ziele ökonomisiert" (Dreier 1990: 109).

Demgegenüber steht die Grundüberzeugung der Väter der Sozialen Marktwirtschaft, dass nur dann, wenn es der Marktwirtschaft gelingt, den gesellschaftlichen Anliegen von Freiheit und sozialer Gerechtigkeit zu dienen, diese auch ihre Berechtigung habe (vgl. Müller-Armack 1948a: 103). Wettbewerb ist lediglich Mittel zum Zweck, und, wie Röpke es ausdrückt, nur bei Beachtung der Maximaldosierung heilsam: 


\begin{abstract}
„Die Wahrheit ist eben, dass derselbe Wettbewerb, den wir zur Regulierung einer freien Wirtschaftsordnung voraussetzen, nach allen Seiten an einen Rand stößt, dessen Überschreitung wir nicht wünschen können. Er bleibt eine moralisch und sozial gefährliche Weise des Verhaltens, die nur in einer gewissen Maximaldosierung und mit Dämpfungen und Moderierungen aller Art verteidigt werden kann“ (Röpke 1979a: 189).
\end{abstract}

\title{
5. Zur (wahrscheinlichen) Zukunft der Sozialen Marktwirtschaft
}

Die Finanz- und Wirtschaftskrise hat gezeigt, dass sich selbst überlassene Märkte, verbunden mit fehlerhaften Anreizsystemen und dem primär eigennutzorientierten Verhalten einzelner Wirtschaftsakteure, zu erheblichen Störungen des gesamten Wirtschaftssystems führen können. Als Ursachen der Wirtschaftskrise lassen sich so zum Einen der allzu leichtfertige Verzicht der Nationalstaaten auf die Ausübung ihrer Regulierungskompetenzen und zum Anderen das offensichtliche Bestreben der Wirtschaftsakteure, bestehende Regeln zum eigenen Vorteil zu umgehen, benennen. Beiden Sichtweisen scheint die Annahme zugrunde zu liegen, Wirtschaft könne als ,moralfreier' Raum interpretiert werden, in dem nicht Fair-Play sondern Cleverness und das Ausnutzen von Macht- und Informationsasymmetrien das Handeln bestimmen darf. Letztlich konvergieren beide Ursachen in dem Irrglauben an die Selbststeuerung der Märkte, die nach dem Prinzip der ,unsichtbaren Hand' die egoistischen Interessen der Einzelnen zum Wohle der Gesellschaft wirksam werden lasse. Dieser Irrglaube ist angesichts der Krise der Einsicht gewichen, dass auch das Funktionieren der Weltwirtschaft maßgeblich von einer stabilen Ordnung der Märkte abhängt. Eine Einsicht, die Röpke bereits aus den Krisenerfahrungen der Vergangenheit herleitete:
„Gerade deshalb ist es geboten, von bestimmten Regeln und festen Prinzipien der Wirtschaftspolitik auszugehen und den Bereich der Willkür auf das äußerste zu beschränken. Das Wirtschaftssystem muss sozusagen ein unzerbrechliches Spiel- zeug sein, das nicht ,kaputt zu machen ist ${ }^{6}-$,fool-proof $f^{\star}$, wie der drastische eng- lische Ausdruck lautet" (Röpke 1979b: 309).

Regulative Idee einer sozialen Marktwirtschaft ist der Wille zur verantwortlichen Gestaltung einer menschendienlichen Wirtschaftsordnung (vgl. Müller-Armack 1948b: 195; Röpke 1979a: 23). Wirtschaft, so das Credo aller sozial-marktwirtschaftlichen Theoretiker, ist gestaltbar und muss gestaltet werden:
„Man spricht bisweilen von den dämonischen Kräften der industrialisierten und technisierten Wirtschaft, denen man kaum gewachsen sein könne. Aber diese Kräfte sind zu fassen, wenn die Bedingungen, unter denen sie sich entwickeln können, entsprechend gestaltet werden. Sonst stolpert man über die Wurzeln des Baumes, den man selbst gepflanzt hat" (Eucken 1990: 224).

Trotz aller Divergenzen im Detail dürfte sich eine Ordnungspolitik im Sinne der Väter der Sozialen Marktwirtschaft daher deutlich von einer rein an den Bedürfnissen der Wirtschaft orientierten ,Angebotspolitik' unterscheiden. Wesentliches Kennzeichen ist, „(...) dass Erhards Ordnungspolitik nicht wie die Angebotspolitik nur ökonomisch begründet ist, sondern auch soziologische, sozialethische, anthropologische und sozialpsychologische Gesichtspunkte beinhaltet" (Wünsche 2001: 63). Sicherlich gilt 
es, die spezifischen historischen Bedingungen, denen sich die Regierung der jungen Bundesrepublik Deutschland und namentlich ihr erster Wirtschaftsminister Ludwig Erhard bei der ,Einführung` der Sozialen Marktwirtschaft gegenüber sah, bei einer historischen Bewertung zu berücksichtigen. So lässt sich diese breite Perspektive der Sozialen Marktwirtschaft - ohne dies hier im Detail diskutieren zu können - auch im Sinne der Konsensbemühungen der jungen Republik um ihre Wirtschaftsordnung interpretieren. Dennoch muss mit Horst Friedrich Wünsche in den Folgejahren eine zunehmende Abkehr insbesondere von den im Konzept der Sozialen Marktwirtschaft stets auch mitgedachten Forderungen nach sozialem Ausgleich und Chancengerechtigkeit in der realen Wirtschaftspolitik der Bundesrepublik konstatiert werden.

„Die neue Wirtschaftspolitik entzog der Synthese von Wirtschafts- und Sozialpolitik die Grundlage, indem sich die Wirtschaftspolitik von sozialen Zielen emanzipierte und sich auf das Ökonomische, vor allem auf Wirtschaftswachstum und Konjunkturstabilisierung, konzentrierte“" (Wünsche 2001: 111).

So bezogen sich die zahlreichen Reformbestrebungen zur Fortschreibung ,der Sozialen Marktwirtschaft ${ }^{`}$ in den Folgejahren vor allem auf die Interpretation der,wirtschaftlichen Rahmenordnung' als Voraussetzung für wirtschaftliches Wachstum. Angesichts der Tatsache, dass dabei hinsichtlich der Frage ,wie viel Staat' und ,wie viel Freiheit' auch die Meinungen der ,geistigen Väter ${ }^{`}$ der Sozialen Marktwirtschaft durchaus unterschiedlich waren, lässt sich jedoch auch bei den politischen Reformbemühungen die Frage nach der ,orthodoxen“ Lesart der Sozialen Marktwirtschaft kaum beantworten. So lässt sich sowohl die Position der ,Marktskeptiker', die den im Zuge der wirtschaftlichen Globalisierung erfolgten Deregulierungen ablehnend gegenüberstehen und ein ,Mehr' an staatlicher Ordnung fordern, als auch die Position der ,Marktbefürworter', die vor allem für ein ,Mehr' an ökonomischen Freiheitsrechten und die Eigenverantwortung der Wirtschaftsakteure eintreten, anhand der unterschiedlichen Schriften zur Sozialen Marktwirtschaft rechtfertigen (vgl. Aßländer/Ulrich 2009: 14f.). Vergessen wird dabei jedoch, dass beide Positionen die moralische Dimension ihrer Forderungen unterschätzen. Beide Positionen rekurrieren stillschweigend auf zwei wesentliche Voraussetzungen der Sozialen Marktwirtschaft: Solidarität und Subsidiarität. Gerade angesichts weltweiter Liberalisierungs- und Deregulierungstendenzen gilt es daher auch, die grundlegenden moralischen Werte einer sozialen Marktwirtschaft erneut zu hinterfragen. Dies betrifft vor allem die mit dem Wirtschaften verbundenen sozialen und ethischen Ziele, wie Freiheit, soziale Gerechtigkeit oder Verantwortung. Wenn, so auch eine der Erkenntnisse der Vordenker der Sozialen Marktwirtschaft, Wirtschaft ohne moralischen Ordnungsrahmen nicht in der Lage ist, zum Wohle der Gesellschaft oder der Gesellschaften zu wirken, kommt der Frage nach den Möglichkeiten einer erneuten, Wertorientierung' der globalen Wirtschaft eine zentrale Rolle zu.

Jedoch stellt sich die Frage, ob ein Konzept, wie es von den Vätern der Sozialen Marktwirtschaft angedacht war, auf die Ordnung der internationalen Märkte angewandt werden kann? Dies hängt letztlich davon ab, ob es der internationalen Politik gelingen wird, sich über drei elementare Voraussetzungen der Wettbewerbsordnung zu verständigen. So muss zum Ersten ein verbindlicher internationaler Rechtsrahmen geschaffen werden, der es erlaubt, das Primat der Politik über die Wirtschaft aufrecht- 
zuerhalten und die (Finanz-)Wirtschaft im Sinne ihrer gesellschaftlichen Aufgabe zu steuern. Zum Zweiten, und dies scheint die schwierigste Aufgabe zu sein, gilt es, sich auf zentrale gemeinsame Werthaltungen zu verständigen, die für eine internationale Wirtschafts- und Gesellschaftspolitik handlungsleitend sein sollen. Schließlich gilt es drittens zu klären, welche Verantwortung den Wirtschaftsakteuren innerhalb dieser Wirtschaftsordnung zukommen soll und wie sich ihr regelkonformes Verhalten sicherstellen lässt.

Im Sinne einer so zu gestaltenden internationalen Ordnung und eingedenk des für das Prinzip der Sozialen Marktwirtschaft konstitutiven Grundgedankens der Subsidiarität plädiert Röpke für eine föderalistische Ordnung der Weltwirtschaft (vgl. Röpke 1979d: 69):

„Einerseits sind wir mit uns darüber im Reinen, dass eine übernationale Organisation eine Notwendigkeit geworden ist. Andererseits aber werden die meisten darin übereinstimmen, dass uns der Gedanke an eine internationale Organisation, die die einzelnen Nationen zu bloßen Verwaltungsbezirken degradiert, noch unerträglicher ist als das bisherige Nebeneinander souveräner Nationen. Weder wollen wir die latente oder offene Anarchie der Nationen, die keinem absolut bindenden und durchsetzbaren Recht unterworfen sind, noch eine Civitas Maxima kontinentaler oder planetarischer Art. Was wir wünschen, ist offenbar, dass sowohl das Eigenleben der Nationen wie die internationale Gemeinschaft zu ihrem Rechte kommen. Keines von beiden soll auf Kosten des anderen wuchern; jedes soll vielmehr dem anderen die Waage halten“ (Röpke 1979d: 67f.).

Dabei bleibt die Aufrechterhaltung auch einer internationalen Ordnung jedoch stets abhängig von der Regelbefolgung der Akteure. Nicht zuletzt kommt so der gesellschaftlichen Vermittlung von Werten eine zentrale Bedeutung zu:

„Wenn man hingegen, wie wir es tun, die Eifersuchtskonflikte systematisch aufheizt, um das Betriebsklima einer ,Konsumgesellschaft' herzustellen, sind früher oder später moralische Desorientierung und psychische Inflation die Folge“ (Sloterdijk 2009).

\section{Literaturverzeichnis}

Aßländer, M. S. (2009a): Das „Adam Smith Problem“ der Sozialen Marktwirtschaft, in: Forum Wirtschaftsethik, Jg. 17/Heft 2, 33-45.

Aßländer, M. S. (2009b): Wohlstand für alle? Die Soziale Marktwirtschaft vor der globalen Herausforderung, in: Aßländer, M. S./Ulrich, P. (Hrsg.): 60 Jahre Soziale Marktwirtschaft - Illusionen und Reinterpretationen einer ordnungspolitischen Integrationsformel, Bern/ Stuttgart/ Wien: Haupt, 223-255.

Aßländer, M. S./ Ulrich, P. (2009): Das Soziale der Sozialen Marktwirtschaft: Impressionen Illusionen - Interpretationen, in: dies. (Hrsg.): a. a. O., 9-25.

Baltzer, S./ Rubkamp, S. (2009): Island mag noch nicht zahlen, 10.02.2009. http://www.faz. net/s/Rub58241E4DF1B149538ABC24D0E82A6266/Doc E2031B243D08644C5A F4802A0824D4C8C ATpl Ecommon Scontent.html, Zugriff am: 23.06.2009. 
Barry, N. P. (1989): Political and Economic Thought of German Neo-Liberals, in: Peacock, A./ Willgerodt, H. (Hrsg.): German Neo-Liberals and the Social Market Economy, London: Macmillan, 105-124.

Bloss, M. et al. (2009): Von der Subprime-Krise zur Finanzkrise - Immobilienblase: Ursachen, Auswirkungen, Handlungsempfehlungen, München: Oldenbourg.

Blum, U. (2009): Deutsche Einheit und Globalisierung als Herausforderungen an die Soziale Marktwirtschaft, in: Aßländer, M. S./ Ulrich, P. (Hrsg.): a. a. O., 175-194.

Bundesverband Deutscher Banken (2009): Finanzmarktkrise: Ursachen und Hintergründe, http:/ / www.bankenverband.de/print.asp?artid=2637\&channel=133810, Zugriff am: 17.04.2009.

Cohan, W. D. (2008): The rise and fall of Jimmy Cayne, in: Fortune, http:// money.cnn.com/2008/07/31/magazines/fortune/rise_and_fall_Cayne_coha n.fortune/inde x.htm, Zugriff am: 18.04.2009.

Deutschmann, Ch. (2008): Die Finanzmärkte und die Mittelschicht: der kollektive BuddenbrocksEffekt, in: Leviathan, Jg. 36/Heft 4, 501-517.

Dreier, W. (1990): Katholische Soziallehre und die Praxis der Sozialen Marktwirtschaft, in: Henning, K./ Bitzer, A. (Hrsg.): Ethische Aspekte von Wirtschaft und Arbeit, Mannheim: Wissenschaftsverlag, 101-122.

Endres, A. (2007): Aktienmärkte - Einstürzende Luftbauten, in Zeit online, http:// images.zeit.de/text/online/2007/11/Boerse-Kurssturz-Immobilienkrise, Zugriff am 17.04.2009.

Eucken, W. (1949): Die Wettbewerbsordnung und ihre Verwirklichung, in: Eucken, W./ Böhm, F. (Hrsg.): Ordo - Jahrbuch für die Ordnung von Wirtschaft und Gesellschaft, Würzburg: ,Journalfranz" Arnulf Liebing OHG.

Eucken, W. (1989): Die Grundlagen der Nationalökonomie, 9. Aufl., Berlin: Springer.

Eucken, W. (1990): Grundsätze der Wirtschaftspolitik, 6. Aufl., Tübingen: J. C. B. Mohr.

Gerardi, K. et al. (2008): Making Sense of the Subprime Crisis, in: Brookings Papers on Economic Activity, Fall 2008.

Habisch, A. (2001): Soziale Marktwirtschaft im Angesicht der Globalisierung, in: Schlecht, O./ Stoltenberg, G. (Hrsg.): Soziale Marktwirtschaft - Grundlagen, Entwicklungslinien, Perspektiven, Freiburg i. Br.: Herder, 193-228.

Hauser, S. (2007): Die ökonomische und soziale Dimension der Sozialen Marktwirtschaft Komplementarität versus Konflikt, in: Hauff M. v. (Hrsg.): Die Zukunftsfähigkeit der Sozialen Marktwirtschaft, Marburg: Metropolis, 71-97.

Heine, S. (2007): Die US Subprime Hypotheken-Krise, http://www.uni-koeln.de/wisofak/bankseminar/ws0708/1223_invbank/WGZBANK_zur_Subprime-

Krise_20070910_kurz.pdf, Zugriff am: 17.04.2009.

Hengsbach, F. (2009): Soziale Marktwirtschaft im Sog des Finanzkapitalismus, in: Aßländer, M. S./ Ulrich, P. (Hrsg.): a. a. O., 291-314.

Hübinger, B. (2001): Rückbesinnung auf die Leitgedanken der Sozialen Marktwirtschaft in den achtziger und neunziger Jahren, in: Schlecht, O./ Stoltenberg, G. (Hrsg.): a. a. O., 115192.

Igel, C. / Wille, E. (2007): Stabilisierung sozialer Sicherungssysteme, insbesondere der Krankenund Pflegeversicherung, in: Hauff M. v. (Hrsg.): a. a. O., 241-282.

Kloten, N. (1989): Role of the Public Sector in the Social Market Economy, in: Peacock, A./ Willgerodt, H. (Hrsg.): a. a. O., 67-104. 
Klump, R. (2001): Soziale Marktwirtschaft: Geistige Grundlagen, ethischer Anspruch, historische Wurzeln, in: Schlecht, O./ Stoltenberg, G. (Hrsg.): a. a. O., 17-59.

Lenel, H. O. (1971): Haben wir noch eine Soziale Marktwirtschaft? in: Böhm, F./ Lutz, F. A./ Meyer, F. W. (Hrsg.): Ordo - Jahrbuch für Wirtschaft und Gesellschaft, Düsseldorf: Küpper, 29-47.

Lenel, H. O. (1989): Evolution of the Social Market Economy, in: Peacock, A./ Willgerodt, H. (Hrsg.): a. a. O., 16-39.

Lin-Hi, N./ Suchanek, A. (2009): Eine wirtschaftsethische Kommentierung der Finanzkrise, in: Forum Wirtschaftsethik, Jg. 17/Heft 1, 20-27.

Maier-Rigaud, F. P./ Maier-Rigand, G. (2001): Das neoliberale Projekt, Marburg: Metropolis.

Maier-Rigand, F. P./ Maier Rigand, G. (2009): Rüstows Konzept der Sozialen Marktwirtschaft Sozial- und Wettbewerbspolitische Dimensionen einer überwirtschaftlichen Ordnung, in: Aßländer, M. S./ Ulrich, P. (Hrsg.): a. a. O., 69-94.

Möschel, W. (1989): Competition Policy from Ordo Point of View, in Peacock, A./ Willgerodt, H. (Hrsg.): a. a. O., 142-159.

Müller-Armack, A. (1948a): Wirtschaftslenkung und Marktwirtschaft, 2. Aufl., Hamburg: Verlag für Wirtschaft und Sozialpolitik.

Müller-Armack, A. (1948b): Das Jahrhundert ohne Gott. Münster: Regensbergsche Verlagsbuchhandlung.

Müller-Armack, A. (1962): Das gesellschaftspolitische Leitbild der Sozialen Marktwirtschaft, in: Institut für Wirtschaftspolitik an der Universität Köln (Hrsg.): Wirtschaftspolitische Chronik. Köln.

Müller-Armack, A. (1972): Die Soziale Marktwirtschaft und ihre Widersacher, in: Erhard, L./ Müller-Armack, A. (Hrsg.): Soziale Marktwirtschaft - Ordnung der Zukunft, Frankfurt am Main/ Berlin/ Wien: Ullstein, 21-40.

Müller-Armack, A. (1976): Wirtschaftsordnung und Wirtschaftspolitik, 2. Aufl., Bern/ Stuttgart: Haupt.

Münchau, W. (2006): Das Ende der Sozialen Marktwirtschaft, München: Hanser.

Münchau, W. (2008): Kernschmelze im Finanzsystem, München: Hanser.

Pies, I. (2001): Eucken und Hayek im Vergleich, Tübingen: J. C. B. Mohr.

Pies, I. (2002): Theoretische Grundlagen demokratischer Wirtschafts- und Gesellschaftspolitik - Der Beitrag Walter Euckens, in: Pies, I./ Leschke, M. (Hrsg.): Walter Euckens Ordnungspolitik, Tübingen: J. C. B. Mohr, 1-35.

Quaas, F. (2009): Der Wirtschaftsstil der Sozialen Marktwirtschaft aus evolutorischer Perspektive, in: in Aßländer, M. S./ Ulrich, P. (Hrsg.): a. a. O., 119-145.

Röpke, W. (1979a): Jenseits von Angebot und Nachfrage, 5. Aufl., Bern/ Stuttgart: Haupt.

Röpke, W. (1979b): Die Gesellschaftskrisis der Gegenwart, 6. Aufl., Bern/ Stuttgart: Haupt.

Röpke, W. (1979c): Civitas humana, 4. Aufl., Bern/ Stuttgart: Haupt.

Röpke, W. (1979d): Internationale Ordnung, 3. Aufl., Bern/ Stuttgart: Haupt.

Röpke, W. (1994): Die Lehre von der Wirtschaft, 13. Aufl., Bern/ Stuttgart/Wien: Haupt.

Schefold, B. (2004): Beiträge zur ökonomischen Dogmengeschichte, Düsseldorf: Verlag für Wirtschaft und Finanzen.

Schmidt, A. (2008): Soziale Marktwirtschaft im Wettbewerb der Systeme, in: Blum, U./ Oberreuter, H. (Hrsg.): 60 Jahre Soziale Marktwirtschaft in einer globalisierten Welt, Sonderheft des Instituts für Wirtschaftsforschung Halle, 95-114. 
Schmidt, A. (2009): Soziale Marktwirtschaft im Zeitalter des Systemwettbewerbs, in: Aßländer, M. S./ Ulrich, P. (Hrsg.): a. a. O., 195-222.

Sesselmeier, W. (2007): Die Zukunft des kontinentaleuropäischen Wohlfahrtsstaates, in: Hauff M. v. (Hrsg.): a. a. O., 99-141.

Sinn, H.-W. (2010a): Kasino-Kapitalismus: Wie es zur Finanzkrise kam, und was jetzt zu tun ist, Frankfurt am Main/ Berlin/ Wien: Ullstein.

Sinn, H.-W. (2010b): „Die Banken wollen nichts ändern“, Interview, in: Handelsblatt, http:/ / www.handelsblatt.com/politik/konjunktur-nachrichten/hans-werner-sinn-diebanken-wollen-nichts-aendern;2571119, Zugriff am: 24.10.2010.

Sloterdijk, P. (2009): „Weltverschwörung der Spießer“, Interview mit Frank A. Meyer, in: Cicero - Magazin für politische Kultur, http://www.manager-magazin.de/unternehmen/ artikel/ 0,2828,611768-3,00.html, Zugriff am: 23.06.2009.

Staatsvertrag (1990): Vertrag über die Schaffung einer Währungs-, Wirtschafts- und Sozialunion zwischen der Bundesrepublik Deutschland und der Deutschen Demokratischen Republik (Staatsvertrag) vom 18. Mai 1990, Presse- und Informationsamt der Bundesregierung Nr. 63 vom 18.5.1990.

Starbatty, J. (2002): Ordoliberalismus, in: Issing. O. (Hrsg.): Geschichte der Nationalökonomie, 4. Aufl., München: Franz Vahlen, 251-270.

Stöttner, R. (2009): Ursachen der aktuellen Finanzkrise, in: Forum Wirtschaftsethik, Jg. 17/Heft 1, 6-19.

Storbeck, O. (2009): Subprime-Krise. Warum hat fast niemand die Krise kommen sehen?, in: Handelsblatt, http://www.handelsblatt.com/politik/wissenswert/warum-hat-fast-nie mand-die-krise-kommen-sehen;2128897, Zugriff am: 17.04.2009.

Thieme, J. H. (1994): Soziale Marktwirtschaft - Ordnungskonzeption und wirtschaftspolitische Gestaltung, 2. Aufl., München: dtv.

Ulrich, P. (2009): Marktwirtschaft in der Bürgergesellschaft - Die Soziale Marktwirtschaft vor der nachholenden gesellschaftspolitischen Modernisierung, in Aßländer, M. S./ Ulrich, P. (Hrsg.): a. a. O., 349-380.

Willgerodt, H. (2006): Der Neoliberalismus - Entstehung, Kampfbegriff und Meinungsstreit, in: Lenel, H. O. et al. (Hrsg.): Ordo - Jahrbuch für Wirtschaft und Gesellschaft, Stuttgart: Lucius \& Lucius, 47-89.

Willgerodt, H. (2007): Der Staat und die Liberalen, in: Lenel, H. O. et al. (Hrsg.): Ordo - Jahrbuch für Wirtschaft und Gesellschaft, Stuttgart: Lucius \& Lucius, 85-98.

Zinn, K. G. (1992): Soziale Marktwirtschaft - Idee, Entwicklung und Politik der bundesdeutschen Wirtschaftsordnung, Mannheim: B. I. Taschenbuchverlag.

Zinn, K. G. (2009a): Soziale Marktwirtschaft - Fortentwicklung und Niedergang während zweier Generationen, in: Forum Wirtschaftsethik, Jg. 17/Heft 2, 7-19.

Zinn, K. G. (2009b): Überdauert die Soziale Marktwirtschaft? Aufstieg und Niedergang des bundesdeutschen Erfolgsmodells, in: Aßländer, M. S./ Ulrich, P. (Hrsg.): a. a. O., 257 289. 